\section{Modeling, Analysis, and Experimentation of Chaos in a Switched Reluctance Drive System}

\author{
K. T. Chau and J. H. Chen
}

\begin{abstract}
In this brief, modeling, analysis, and experimentation of chaos in a switched reluctance (SR) drive system using voltage pulsewidth modulation are presented. Based on the proposed nonlinear flux linkage model of the SR drive system, the computation time to evaluate the Poincaré map and its Jacobian matrix can be significantly shortened. Moreover, the stability analysis of the fundamental operation is conducted, leading to determine the stable parameter ranges and hence to avoid the occurrence of chaos. Both computer simulation and experimental measurement are given to verify the theoretical modeling and analysis.
\end{abstract}

Index Terms-Bifurcation, chaos, drive system, stability, switched reluctance (SR) motor.

\section{INTRODUCTION}

Chaos in switching dc-dc converters has been actively investigated for a number of years [1]-[4]. Recently, chaotic behaviors in electric drive systems have also been discussed [5]-[9]. Namely, chaos in an inverter-fed induction drive system using pulsewidth modulation (PWM) was investigated by numerical simulation [5]; a strange attractor in an idealized brushless dc drive system was modeled by using a Lorenz equation [6]; chaos in dc drive systems was modeled, analyzed, and experimented [7], [8]; and chaos in a switched reluctance (SR) drive system was preliminarily investigated by computer simulation [9].

Among various modern drive systems, the SR drive system has been identified to have promising industrial applications [10]. It takes the advantages of high efficiency, high power density, high reliability, and low manufacturing cost, but suffers from the drawbacks of high nonlinearities and control complexity [11]. Thus, it is anticipated that the SR drive system is more prone to chaos. The purpose of this brief is to first present the modeling, analysis, and experimentation of chaos in an SR drive system using voltage PWM regulation. To the best of the authors' knowledge, the investigation of chaos in electric drive systems was covered only in a few studies [5]-[9], and the experimental confirmation of chaos in the SR drive system was absent in literature.

\section{SYSTEM MODELING}

Fig. 1 shows a typical three-phase SR motor drive for exemplification. The corresponding speed control is achieved by applying PWM chopping to its motor voltages. The commutation strategy uses rotor position feedback to select the turn-on angle $\theta_{o}$ and turn-off angle $\theta_{c}$ of those lower-leg power switches $\left(A_{2}, B_{2}\right.$, and $\left.C_{2}\right)$. When the phase windings are conducted in turn, the dwell interval $\theta_{d}=\theta_{c}-\theta_{o}$ of each phase winding is selected to be equal to the commutation angle $\theta_{s}=2 \pi /\left(m N_{r}\right)$, where $m$ is the number of phases, and $N_{r}$ is the number of rotor poles. As shown in Fig. 2, the stator phase A winding starts to conduct at $\theta=\theta_{o}$ and ends at $\theta=\theta_{o}+\theta_{s}$. Subsequently, the phase $\mathrm{B}$ winding conducts from $\theta=\theta_{o}+\theta_{s}$ to $\theta=\theta_{o}+2 \theta_{s}$, and

Manuscript received October 23, 2000; revised June 21, 2001. This work was supported by the Research Grants Council, Hong Kong Special Administrative Region, China, under Project HKU 7128/9 9E. This paper was recommended by Associate Editor D. Czarkowski.

K. T. Chau is with the Department of Electrical and Electronic Engineering, The University of Hong Kong, Hong Kong, China.

J. H. Chen is with the Department of Electrical Engineering, Tsinghua University, Beijing 100875, China.

Digital Object Identifier 10.1109/TCSI.2003.811030 the phase $\mathrm{C}$ winding conducts from $\theta=\theta_{o}+2 \theta_{s}$ to $\theta=\theta_{o}+3 \theta_{s}$. For each cycle of conduction of all phase windings, the rotating angle of the rotor is a total of $2 \pi / N_{r}$. Fig. 2(a) also indicates that each phase winding conducts at the instant of decreasing magnetic reluctance between the stator and rotor, hence producing a positive torque to drive the rotor. For the sake of synchronizing the voltage PWM regulation with the phase commutation, the ramp voltage $v_{r}$ for each phase winding is a function of the instantaneous rotor displacement $\theta$

$$
v_{r}=v_{l}+\left(v_{u}-v_{l}\right) \frac{\left[\left(\theta-\theta_{o}\right) \bmod \theta_{T}\right]}{\theta_{T}}
$$

where $v_{l}$ and $v_{u}$ are the lower and upper bounds of the ramp voltage, $\theta_{T}=\theta_{d} / n_{\theta}$ is its period, $n_{\theta}$ is an integer, and $\left(\theta-\theta_{o}\right) \bmod \theta_{T}$ is defined as the remainder of $\left(\theta-\theta_{o}\right)$ divided by $\theta_{T}$. As shown in Fig. 1, since $v_{\omega}$ and $v_{\omega}^{*}$ are linear functions of the instantaneous speed $\omega$ and reference speed $\omega^{*}$, respectively, the speed control signal $v_{c}$ can be expressed as

$$
v_{c}=g\left(\omega-\omega^{*}\right)
$$

where $g$ is the overall feedback gain incorporating both the F/V converter and the op-amp OA. Then, both $v_{r}$ and $v_{c}$ are fed into the comparator CM which outputs the signal to turn on or off those upper-leg power switches $\left(A_{1}, B_{1}\right.$ and $\left.C_{1}\right)$, depending on the phase commutation. When $v_{c}$ exceeds $v_{r}$, the upper-leg switch is the same phase of the turn-on lower-leg switch, which is off; otherwise, it is on. The other phase switches remain off. The corresponding waveforms of $v_{c}$ and $v_{r}$, as well as switching signals, are shown in Fig. 2(b).

Instead of the phase current $i_{k}$, the phase flux linkage $\psi_{k}$ is chosen as the state variable so that the system differential equation does not involve the calculation of $\partial \psi_{k}\left(\theta, i_{k}\right) / \partial \theta$ and $\partial \psi_{k}\left(\theta, i_{k}\right) / \partial i_{k}$. Since $m$ phase windings of the SR motor are conducted in turn, only two adjacent phase windings have currents at the same time when $m>2$. For the sake of clarity and simplicity, $m$-phase windings conducted in turn are represented by only two-phase windings (namely 1 and 2, activated alternately). When the phase winding is controlled by PWM regulation, it is called activated winding; otherwise, it is called inactivated winding. Considering the winding two lags behind the winding 1 with $\theta_{s}$, the system equation of the SR drive can be expressed as

$$
\begin{aligned}
&\left\{\begin{array}{l}
\frac{d \theta}{d t}=\omega \\
\frac{d \omega}{d t}=\frac{\left(-B \omega+T_{e}\left(\theta, \psi_{1}, \psi_{2}, \theta_{s}\right)-T_{l}\right)}{J} \\
\frac{d \psi_{1}}{d t}=-R i_{1}\left(\theta, \psi_{1}\right)+u_{1}(\theta) \\
\frac{d \psi_{2}}{d t}=-R i_{2}\left(\theta-\theta_{s}, \psi_{2}\right)+u_{2}\left(\theta-\theta_{s}\right)
\end{array}\right. \\
& T_{e}=-\frac{\partial}{\partial \theta} \int_{0}^{\psi_{1}} i_{1}(\theta, \psi) d \psi-\frac{\partial}{\partial \theta} \int_{0}^{\psi_{2}} i_{2}\left(\theta-\theta_{s}, \psi\right) d \psi \\
& u_{k}= \begin{cases}V_{s} \varepsilon\left(v_{r}(\theta)-v_{c}\right), & \left(\theta \bmod 2 \theta_{s}\right) \in\left[\theta_{o}, \theta_{o}+\theta_{s}\right) \\
-V_{s} \varepsilon\left(\psi_{k}\right), & \left(\theta \bmod 2 \theta_{s}\right) \in\left[\theta_{o}+\theta_{s}, \theta_{o}+2 \theta_{s}\right)\end{cases}
\end{aligned}
$$

where $u_{k}$ is the phase voltage, $V_{s}$ is the dc supply voltage, $R$ is the phase resistance, $B$ is the viscous damping, $J$ is the load inertia, $T_{e}$ is the electromagnetic torque, $T_{l}$ is the load torque, and $\varepsilon$ is the unit step function. Because of its high nonlinearity, $\psi_{k}\left(\theta, i_{k}\right)$ is approximated by a series of two-dimensional (2-D) quadratic Lagrange interpolation functions of $\theta$ and $i_{k}$. These 2-D grids are formulated by using the manufacturer's design data or by employing finite element analysis of 


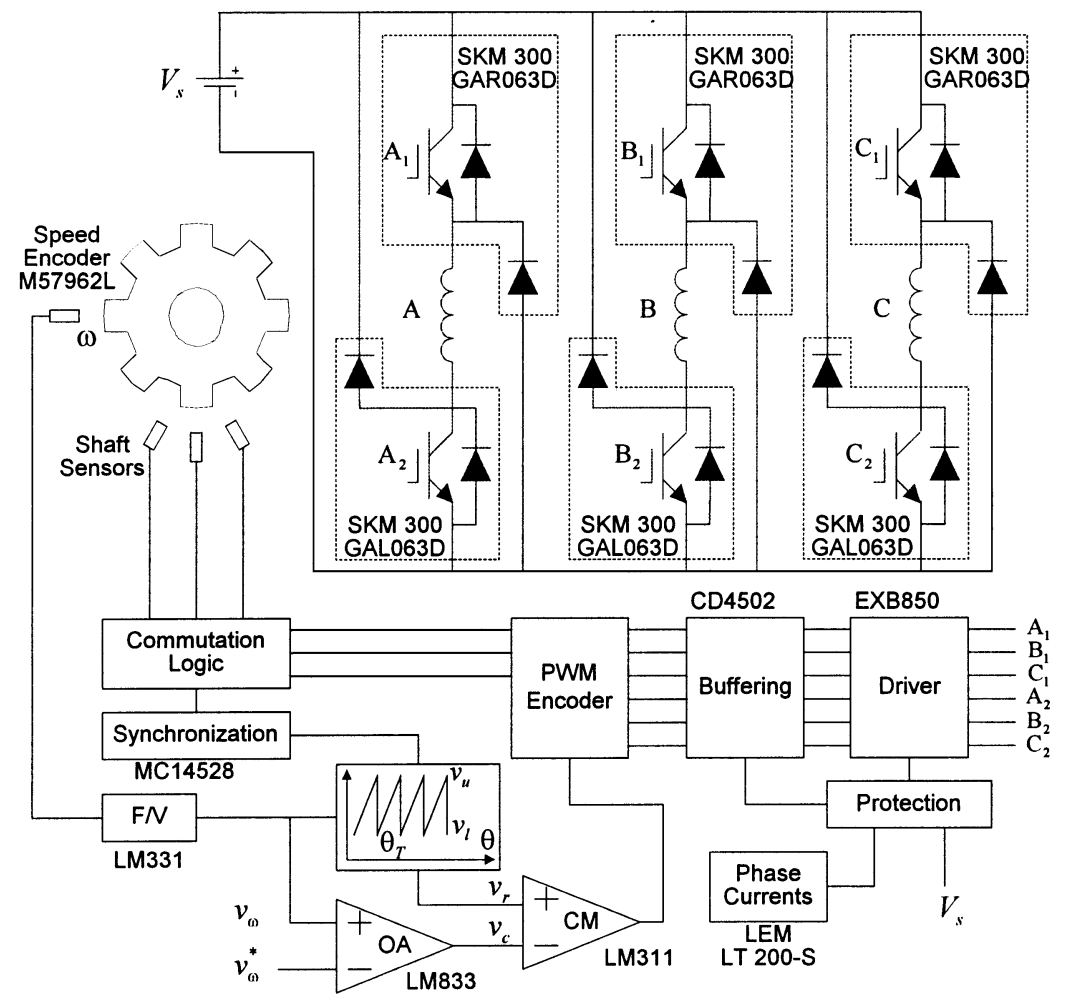

Fig. 1. Schematic diagram of SR motor drive.

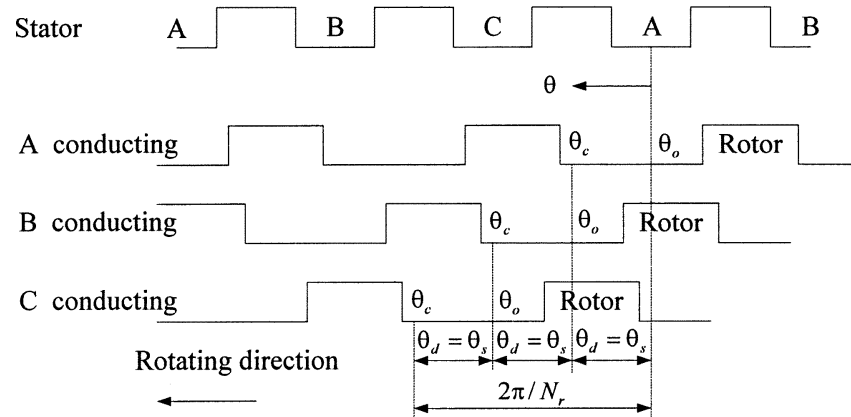

(a)
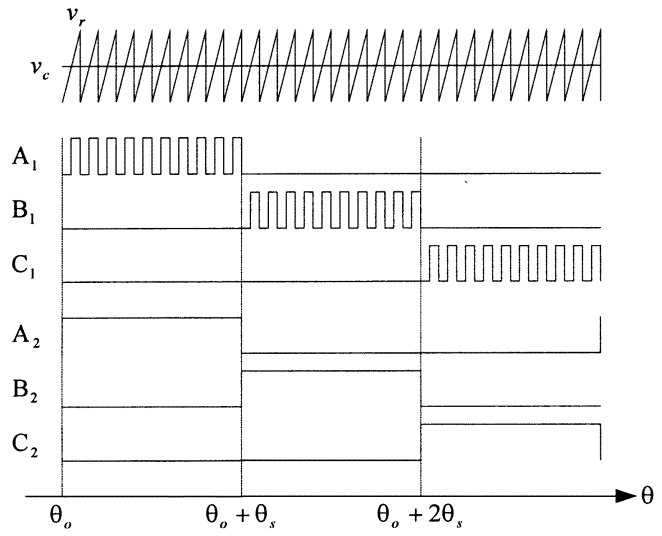

(b)

Fig. 2. Operation principle of SR motor drive. (a) Rotor positions. (b) Switching signals.

the SR motor. This approach can significantly reduce the complexity in the calculation of flux linkages. Moreover, $i_{k}\left(\theta, \psi_{k}\right)$ can similarly be obtained by numerical inversion of the relations of $\psi_{k}\left(\theta, i_{k}\right)$. Since the current is much easier to be measured than the flux linkage, it is chosen as the output variable. By defining the state vector as $\mathbf{X}=$ $\left(\theta, \omega, \psi_{1}, \psi_{2}\right)^{T}$, and the output vector as $\mathbf{Y}=(\theta, \omega, i)^{T}$, where $i=$ $i_{1}+i_{2}$, the system equation given by (3) can be rewritten as

$$
\left\{\begin{array}{l}
\dot{\mathbf{X}}=f_{t}(\mathbf{X}) \\
\mathbf{Y}=M_{t}(\mathbf{X})
\end{array}\right.
$$

\section{Modeling OF CHAOS}

In order to construct the Poincaré map, a hyperplane $\Sigma \in \Re^{3}$ is defined as

$$
\Sigma:=\left\{\mathbf{X}:\left[\left(\theta-\theta_{o}\right) \bmod \theta_{s}\right]=0\right\} .
$$

The trajectory of $\mathbf{X}$ under observation repeatedly passes through $\Sigma$ when $\theta$ increases monotonically. Thus, the sequence of $\Sigma$ crossing defines a Poincaré map $P: \Re^{3} \times \Re^{3}$ as given by

$$
\left(\omega, \psi_{1}, \psi_{2}\right)_{n+1}^{T}=P\left(\left(\omega, \psi_{1}, \psi_{2}\right)_{n}^{T}\right) .
$$

Actually, the solution of this map, the so-called orbit [12], is a sequence of samples at the turn-on angle of each phase winding. In order to avoid the calculation of $\Sigma$ crossing, the rotor displacement $\theta$, rather than time $t$, is selected as the independent variable of the system equation given by (3). The next crossing of the plane $\theta_{n+1}=\theta_{o}+(n+1) \theta_{s}$ can be directly calculated by integrating from $\theta_{n}=\theta_{o}+n \theta_{s}$ to $\theta_{n+1}$. To make $\theta$ an independent variable, (3) is expressed as

$$
\left\{\begin{array}{l}
\frac{d \omega}{d \theta}=\frac{\left(-B \omega+T_{e}\left(\theta, \psi_{1}, \psi_{2}, \theta_{s}\right)-T_{l}\right)}{(J \omega)} \\
\frac{d \psi_{1}}{d \theta}=\frac{\left(-R i_{1}\left(\theta, \psi_{1}\right)+u_{1}(\theta)\right)}{\omega} \\
\frac{d \psi_{2}}{d \theta}=\frac{\left(-R i_{2}\left(\theta-\theta_{s}, \psi_{2}\right)+u_{2}\left(\theta-\theta_{s}\right)\right)}{\omega} .
\end{array}\right.
$$


Since $u_{k}$ is piecewise continuous in terms of $\theta$, it is much easier to find out the discontinuity points of $u_{k}$ in (9) than in (3). Accordingly, (9) can be separately solved within different continuous intervals. Hence, it is much more efficient to use (9) to calculate the Poincaré map than the use of (3). By redefining the state vector as $\mathbf{X}(\theta)=$ $\left(\omega, \psi_{1}, \psi_{2}\right)^{T}$ and the output vector as $\mathbf{Y}(\theta)=(\omega, i)^{T}$, (9) can be rewritten as

$$
\left\{\begin{array}{l}
\dot{\mathbf{X}}=f_{\theta}(\mathbf{X}, \theta) \\
\mathbf{Y}=M_{\theta}(\mathbf{X})
\end{array}\right.
$$

The Poincaré map (8) can also be rewritten as

$$
\left\{\begin{array}{l}
\mathbf{X}_{n+1}=P\left(\mathbf{X}_{n}\right) \\
\mathbf{Y}_{n}=M_{\theta}\left(\mathbf{X}_{n}\right)
\end{array}\right.
$$

Although $f_{\theta}$ is piecewise continuous, the solution of (10) is continuous, and hence, $P$ is also continuous. It should be noted that the map $M_{\theta}$ in (10) is a noninvertible map within the whole set of the solution. For example, if $\psi_{1}$ and $\psi_{2}$ are simultaneously nonzero, $\mathbf{X}$ is a multivalued function of $\mathbf{Y}$. However, the map $M_{\theta}$ in (11) is homeomorphism, namely $M_{\theta}$ and $M_{\theta}^{-1}$ are continuous, because one of $\psi_{1}$ and $\psi_{2}$ of $\mathbf{X}_{n}$ is zero. It results that a new map $Q: \mathbf{Y}_{n+1}=Q\left(\mathbf{Y}_{n}\right)$ can be defined as $Q=M_{\theta} \circ P \circ M_{\theta}^{-1}$. Thus, the maps $P$ and $Q$ are topologically conjugate [13], which means that the corresponding orbits $\left\{\mathbf{X}_{n}\right\}$ and $\left\{\mathbf{Y}_{n}\right\}$ have the same dynamics although they represent different physical variables of the SR drive system. For example, if $\mathbf{X}^{*}$ is a fixed point of $P$, then $\mathbf{Y}^{*}=M_{\theta}\left(\mathbf{X}^{*}\right)$ is also a fixed point of $Q$. The eigenvalues of their Jacobian matrices $D P\left(\mathbf{X}^{*}\right)$ and $D Q\left(\mathbf{Y}^{*}\right)$ are identical. The orbit $\left\{\mathbf{X}_{n}\right\}$ is used to locate the periodic and chaotic orbits, whereas the orbit $\left\{\mathbf{Y}_{n}\right\}$ is used to illustrate the trajectories and waveforms.

The Jacobian matrix $D P\left(\mathbf{X}_{n}\right)$ of the Poincaré map $P$ with respect to $\mathbf{X}_{n}$ is the solution $\mathbf{Z}_{n+1}=\mathbf{Z}\left(\theta_{n+1}\right) \in \Re^{3} \times \Re^{3}$ of the variational equation of its underlying system, as given by

$$
\dot{\mathbf{Z}}(\theta)=\frac{\partial f_{\theta}(\mathbf{X}(\theta), \theta)}{\partial \mathbf{X}} \mathbf{Z}(\theta) \quad \mathbf{Z}\left(\theta_{n}\right)=\mathbf{Z}_{n} .
$$

Considering that the winding 1 is inactivated, it yields

$$
\begin{aligned}
& \frac{\partial f_{\theta}}{\partial \mathbf{X}} \\
= & \left(\begin{array}{ccc}
\frac{-T_{e}+T_{l}}{J \omega^{2}} & \frac{1}{J \omega} \frac{\partial i_{1}}{\partial \theta} & \frac{1}{J \omega} \frac{\partial i_{2}}{\partial \theta} \\
\frac{R i_{1}-u_{1}}{\omega^{2}} & -\delta_{\theta}\left(\theta-\theta_{\psi}\right)-\frac{R}{\omega} \frac{\partial i_{1}}{\partial \psi_{1}} & 0 \\
\frac{1}{\omega} \frac{\partial u_{2}}{\partial \omega}+\frac{R i_{2}-u_{2}}{\omega^{2}} & 0 & -\frac{R}{\omega} \frac{\partial i_{2}}{\partial \psi_{2}}
\end{array}\right)
\end{aligned}
$$

where

$$
\frac{\partial u_{2}}{\partial \omega}=\frac{g V_{s} \delta_{\theta}\left(\theta-\theta_{\omega}\right)}{\frac{g\left(-B \omega\left(\theta_{\omega}\right)+T_{e}\left(\theta_{\omega}\right)-T_{l}\right)}{\left(J \omega\left(\theta_{\omega}\right)\right)-\frac{\left(v_{u}-v_{l}\right)}{\theta_{T}}}}
$$

both $\partial i_{k} / \partial \theta$ and $\partial i_{k} / \partial \psi_{k}$ can be obtained by directly differentiating $i_{k}\left(\theta, \psi_{k}\right), \delta_{\theta}$ is the Dirac delta function that is the derivative of $\varepsilon, \theta_{\psi}$ and $\theta_{\omega}$ are the discontinuity points of $u_{1}$ and $u_{2}$, which can be obtained by solving $\psi_{1}\left(\theta_{\psi}\right)=0$ and $v_{r}\left(\theta_{\omega}\right)-v_{c}\left(\theta_{\omega}\right)=0$, respectively.

For an arbitrary point $\mathbf{X}_{n}$, the corresponding initial value $\mathbf{Z}_{n}$ of the variational equation given by (12) is usually an identity matrix. However, since $\psi_{2}\left(\theta_{n}\right)=0$, it results that $\partial \mathbf{X}_{n} / \partial \psi_{2}=\left(\begin{array}{lll}0 & 0 & 0\end{array}\right)^{T}$. Hence, the third column of the identity matrix $\mathbf{Z}_{n}$ should be replaced by a zero vector.

Actually, the period of $\psi_{1}$ and $\psi_{2}$ of $\mathbf{X}$ is always $2 \theta_{s}$ rather than $\theta_{s}$. In order to attain the period- 1 orbit of $P$, the winding 1 always stands for the inactivated winding at each iteration of $P$, resulting that $\psi_{1}$ and $\psi_{2}$ must exchange their values after each iteration of $P$. Thus, the fixed point $\mathbf{X}^{*}$ of $P$ and its Jacobian matrix are defined as

$$
\begin{aligned}
\mathbf{X}^{*} & =\mathbf{C} P\left(\mathbf{X}^{*}\right) \\
\mathbf{J}_{1} & =\mathbf{C} D P\left(\mathbf{X}^{*}\right)
\end{aligned}
$$

where

$$
\mathbf{C}=\left(\begin{array}{lll}
1 & 0 & 0 \\
0 & 0 & 1 \\
0 & 1 & 0
\end{array}\right)
$$

The fixed point $\mathbf{X}^{*}$ of $P$ can also be located by using the Newton-Raphson algorithm as given by

$$
\mathbf{X}^{(i+1)}=\mathbf{X}^{(i)}-\left(\mathbf{C} D P\left(\mathbf{X}^{(i)}\right)-\mathbf{1}\right)^{-1}\left(\mathbf{C} P\left(\mathbf{X}^{(i)}\right)-\mathbf{X}^{(i)}\right)
$$

where $D P\left(\mathbf{X}^{(i)}\right)$ can be evaluated from (12) and (13). By checking the characteristic multipliers, that is, the eigenvalues of the Jacobian matrix, the stable region of the period-1 orbit for the fundamental operation can readily be obtained.

\section{ANALYSIS OF CHAOS}

In order to assess the proposed modeling of chaos, the corresponding analysis is carried out based on a practical three-phase SR drive system that has been designed for an electric vehicle [11]. The parameter values are $V_{s}=150 \mathrm{~V}, N_{s}=12, N_{r}=8, \theta_{s}=15^{\circ}, \theta_{o}=3.75^{\circ}$, $R=0.15 \Omega, B=0.00075 \mathrm{Nm} / \mathrm{rads}^{-1}, J=0.025 \mathrm{kgm}^{2}, v_{u}=5 \mathrm{~V}$, $v_{l}=1 \mathrm{~V}, n_{\theta}=10, \theta_{T}=1.5^{\circ}, \omega^{*}=50 \mathrm{rad} / \mathrm{s}$, and $T_{1}=8.6 \mathrm{Nm}$.

When $g=1.3 \mathrm{~V} / \mathrm{rads}^{-1}$, the steady-state behavior of the SR drive system is the fundamental operation. The corresponding simulated waveforms of $v_{c}, v_{r}$, and $i$ as well as the simulated phase-plane trajectory of $i$ versus $v_{c}$ are shown in Fig. 3, in which $\theta$ is expressed as the integer multiple of $\theta_{s}$. As shown in Fig. 3(a), there is no skipping cycle during PWM regulation, namely, $v_{c}$ crosses every $v_{r}$. The fluctuation of $v_{c}$ is also small (from 4.0 to $4.4 \mathrm{~V}$ ), and the corresponding $\omega$ is from 53.1 to $53.4 \mathrm{rad} / \mathrm{s}$. As shown in Fig. 3(b), the corresponding $i$ is periodic in terms of $\theta_{s}$, and its fluctuation is from 23 to $60 \mathrm{~A}$. Since $n_{\theta}=10, i$ has ten peaks within each $\theta_{s}$, resulting that the phase-plane trajectory of this periodic solution is a cycle having ten peaks, as shown in Fig. 3(c).

When $g=4.8 \mathrm{~V} / \mathrm{rads}^{-1}$, the SR drive system operates in chaos. The simulated chaotic waveforms and trajectory are shown in Fig. 4. Different from the periodic solution, it has skipping cycles within each $\theta_{s}$ as shown in Fig. 4(a), in which $v_{c}$ is higher than $v_{r}$, and no intersection occurs. Furthermore, the number of skipping cycles within each $\theta_{s}$ is a random-like variable. It follows that the oscillating magnitudes of $v_{c}$ (being the same shape of $\omega$ ) and $i$ are all fluctuating, as shown in Fig. 4(a) and (b), respectively. As expected, Fig. 4(c) shows that the trajectory of $i$ versus $v_{c}$ is a random-like bounded phase portrait. It can be found that even though the fluctuation of $\omega$ is still acceptable (from 50.6 to $51.6 \mathrm{rad} / \mathrm{s}$ ), the fluctuation of $v_{c}$ is severe (from 2.9 to $7.6 \mathrm{~V}$ ), resulting that the fluctuation of $i$ is exceptionally large (from 0 to $120 \mathrm{~A}$ ), which is highly undesirable.

In order to determine the boundary of the stable fundamental operation, all period- 1 orbits are first located by using the Newton-Raphson algorithm in (16). Then, their characteristic multipliers are evaluated by computing the eigenvalues of the corresponding Jacobian matrices obtained from (12)-(15). By drawing the line such that the magnitude of the characteristic multipliers is equal to unity, the stable region of $T_{1}$ versus $g$ for the fundamental operation can be obtained as shown in Fig. 5. In case the characteristic multipliers are complex conjugates while their magnitudes are less than unity, the system is still stable but 


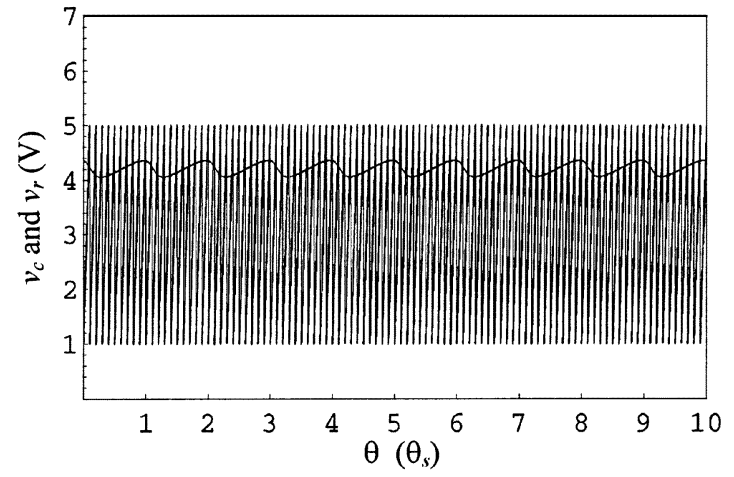

(a)

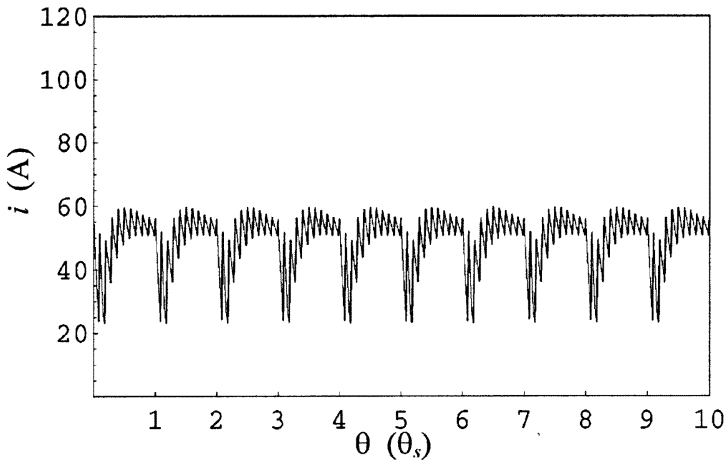

(b)

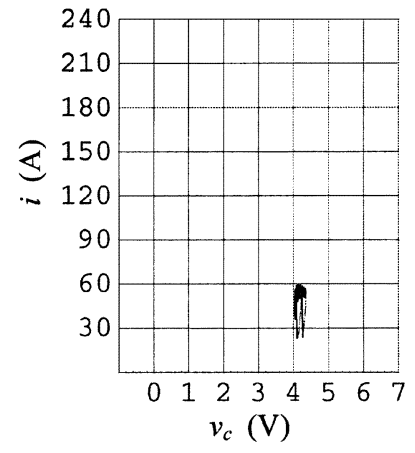

(c)

Fig. 3. Simulated fundamental operation with $g=1.3 \mathrm{~V} / \mathrm{rads}^{-1}$. (a) Control and ramp voltage waveforms. (b) Total current waveform. (c) Trajectory of total current versus control voltage.

spirally converging to the fixed point. The unstable region for the fundamental operation involves both subharmonic and chaotic operations. It should be noted that Fig. 5 is highly desirable for the designer of SR drive systems.

\section{EXPERIMENTATION OF CHAOS}

Based on the same SR drive system, experimentation is conducted. Since $\omega$ has a large dc bias while its variation is relatively small, it is not so clear to assess the speed variation based on the direct measurement of $\omega$. In contrast, $v_{c}$ is the amplified speed error which not only exhibits a clear pattern of speed variation, but is also easily measurable. Thus, $v_{c}$ is measured to represent $\omega$. The measured trajectory and waveforms of $i$ and $v_{c}$ when $g=1.3 \mathrm{~V} / \mathrm{rads}^{-1}$ are shown in Fig. 6. This illustrates that the SR drive system operates in the period-1 orbit, actually the stable fundamental operation. It can be observed that $i$ and $v_{c}$ are not of exact periodicity, which is due to the inevitable imperfections of the

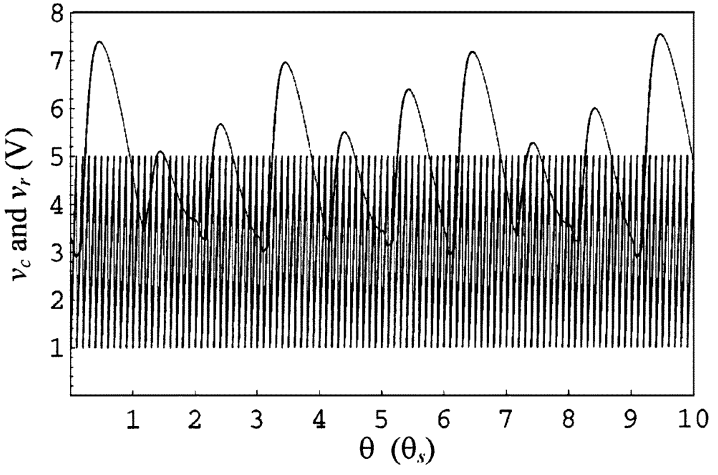

(a)

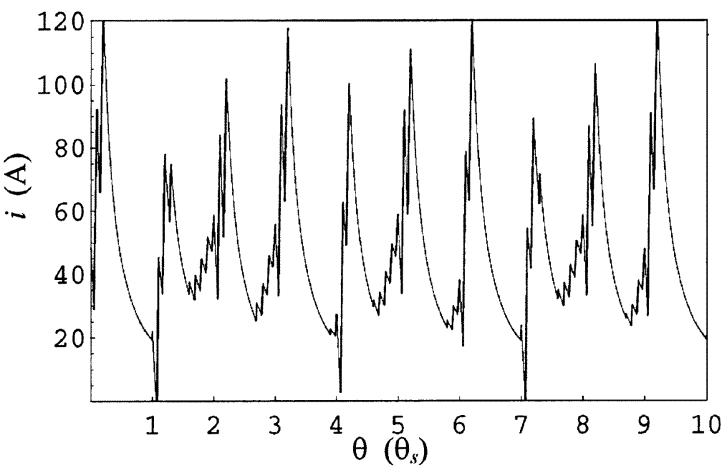

(b)

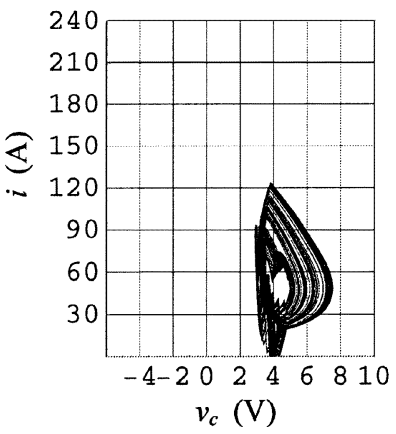

(c)

Fig. 4. Simulated chaotic operation with $g=4.8 \mathrm{~V} / \mathrm{rads}^{-1}$. (a) Control and ramp voltage waveforms. (b) Total current waveform. (c) Trajectory of total current versus control voltage.

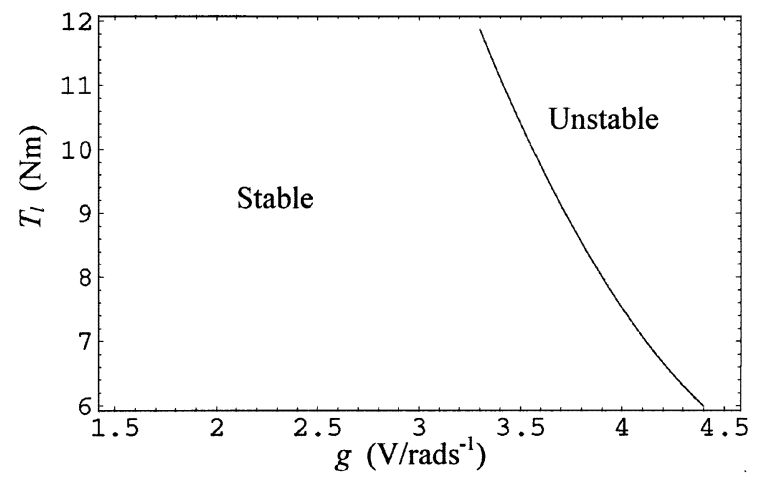

Fig. 5. Stable and unstable regions for fundamental operation. 

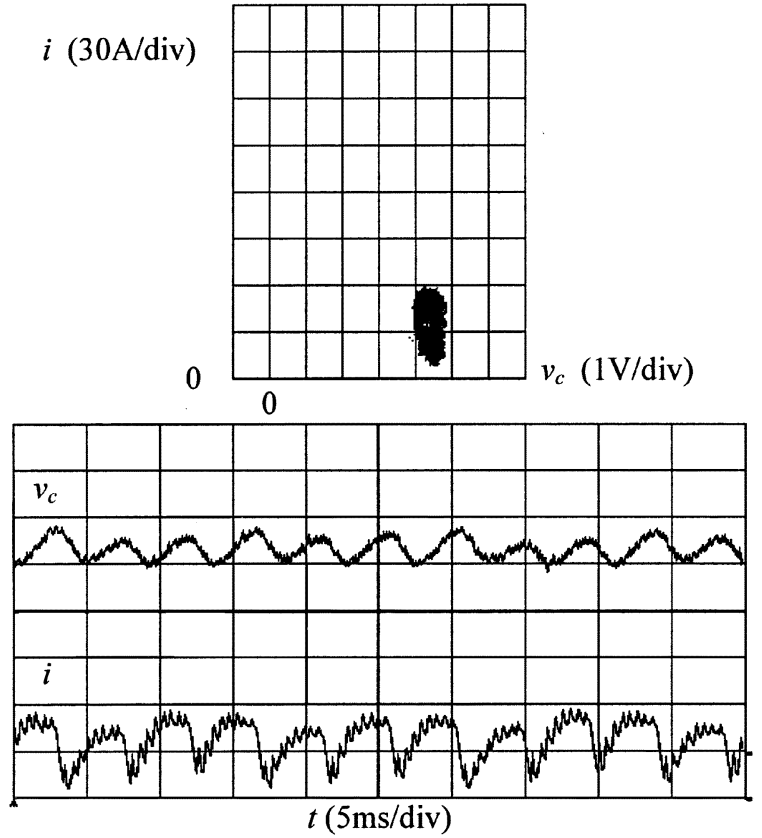

Fig. 6. Measured trajectory and waveforms of feedback control voltage and total current at fundamental operation with $g=1.3 \mathrm{~V} / \mathrm{rads}^{-1}$.
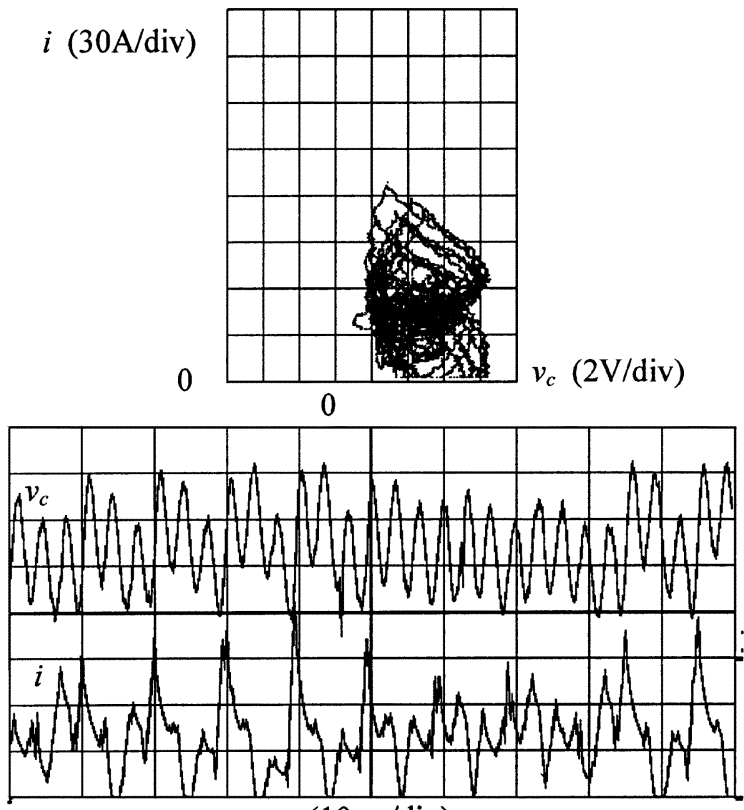

$t(10 \mathrm{~ms} / \mathrm{div})$

Fig. 7. Measured trajectory and waveforms of feedback control voltage and total current at chaotic operation with $g=4.8 \mathrm{~V} / \mathrm{rads}^{-1}$.

practical SR motor drive, such as the mechanical eccentricity of the drive shaft and the torsional oscillation of the coupler. Also, it can be found that $i$ lies roughly between 20 and $60 \mathrm{~A}$, while $v_{c}$ lies between 4.0 and $4.5 \mathrm{~V}$. By comparing these results with the waveforms and trajectory shown in Fig. 3, the measured results and the theoretical prediction have a good agreement.

Moreover, by selecting $g=4.8 \mathrm{~V} / \mathrm{rads}^{-1}$, the measured trajectory and waveforms of $i$ and $v_{c}$ shown in Fig. 7 illustrate that the SR drive system operates in chaos. It can be found that the boundaries of $i$ and $v_{c}$ lie roughly between 0 and $120 \mathrm{~A}$ and between 2 and $8 \mathrm{~V}$, respec- tively. Different from the period-1 orbit in which the measured trajectory and waveforms are directly compared with the theoretical prediction, the chaotic trajectory and waveforms measured in the experiment can hardly match the theoretical ones because the chaotic behavior is aperiodic so that the period of measurement cannot be the same with that of theoretical analysis. Nevertheless, it can be found that the measured boundaries of the chaotic trajectory shown in Fig. 7 resemble the theoretical prediction in Fig. 4, which is actually a property of chaos.

\section{CONCLUSION}

In this brief, the modeling, analysis, and experimentation of chaos in the SR drive system using voltage PWM regulation have been presented. The corresponding experimental confirmation of chaos first appeared in literature. Based on the proposed nonlinear flux linkage model of the SR drive system, the computation time to evaluate the Poincaré map and its Jacobian matrix can be significantly shortened. Also, the stability analysis of the fundamental operation is carried out, which can depict the stable operating region of load torque and feedback gain. The proposed modeling approach and analysis technique can readily be applied or extended to other electric drive systems.

\section{REFERENCES}

[1] J. H. B. Deane, P. Ashwin, D. C. Hamill, and D. J. Jefferies, "Calculation of the periodic spectral components in a chaotic DC-DC converter," IEEE Trans. Circuits Syst. I, vol. 46, pp. 1313-1319, Nov. 1999.

[2] M. di Bernardo and F. Vasca, "Discrete-time maps for the analysis of bifurcations and chaos in DC/DC converters," IEEE Trans. Circuits Syst. I, vol. 47, pp. 130-143, Feb. 2000.

[3] A. Magauer and S. Banerjee, "Bifurcations and chaos in the tolerance band PWM technique," IEEE Trans. Circuits Syst. I, vol. 47, pp. 254-259, Feb. 2000.

[4] C. K. Tse, Y. M. Lai, and H. H. C. Iu, "Hopf bifurcation and chaos in a free-running current-controlled Cuk switching regulator," IEEE Trans. Circuits Syst. I, vol. 47, pp. 448-457, Apr. 2000.

[5] I. Nagy, L. Matakas Jr., and E. Masada, "Application of the theory of chaos in PWM technique of induction motors," in Proc. Int. Power Electron. Conf., 1995, pp. 58-63.

[6] N. Hemati, "Strange attractors in brushless DC motors," IEEE Trans. Circuits Syst. I, vol. 41, pp. 40-45, Jan. 1994.

[7] J. H. Chen, K. T. Chau, and C. C. Chan, "Analysis of chaos in currentmode-controlled DC drive systems," IEEE Trans. Ind. Electron., vol. 47, pp. 67-76, Feb. 2000

[8] J. H. Chen, K. T. Chau, S. M. Siu, and C. C. Chan, "Experimental stabilization of chaos in a voltage-mode DC drive system," IEEE Trans. Circuits Syst. I, vol. 47, pp. 1093-1095, July 2000.

[9] J. H. Chen, K. T. Chau, and Q. Jiang, "Analysis of chaotic behavior in switched reluctance motors using voltage PWM regulation," Elect. Power Compon. Syst., vol. 29, no. 3, pp. 211-227, 2001.

[10] T. J. E. Miller, Switched Reluctance Motors and Their Control. Oxford, U. K.: Magna, 1993.

[11] Y. J. Zhan, C. C. Chan, and K. T. Chau, "A novel sliding mode observer for indirect position sensing of switched reluctance motor drives," IEEE Trans. Ind. Electron., vol. 46, pp. 390-397, Apr. 1999.

[12] T. S. Parker and L. O. Chua, Practical Numerical Algorithms for Chaotic Systems. New York: Springer-Verlag, 1989.

[13] S. Wiggins, Introduction to Applied Nonlinear Dynamical Systems and Chaos. New York: Springer-Verlag, 1990. 